\title{
Matching and oddity learning in pigeons: Effects of penalty time for incorrect responding
}

\author{
VERNON STRENGTH \\ Valparaiso University, Valparaiso, Indiana \\ and \\ THOMAS R. ZENTALL \\ University of Kentucky, Lexington, Kentucky
}

\begin{abstract}
In simultaneous matching-to-sample and oddity-from-sample tasks, briefly delaying the offset of trial stimuli following an incorrect choice response was found to facilitate task acquisition (Experiment 1). Because this penalty-time procedure also resulted in longer choice-response latencies, it was hypothesized that any procedure that increased response latency would facilitate task acquisition. However, in Experiment 2, no evidence of facilitation was found when a 2-sec pause was imposed prior to the choice response. The results of Experiment 3 suggest that penalty-time facilitation of acquisition was not due to either the added differential outcome on correct versus incorrect trials (i.e., incorrect choice responses do not darken the keys as do correct choice responses) or the aversive effects associated with trial prolongation (i.e., incorrect responses not only result in the absence of reinforcement but also delay the start of the next trial). Instead, results suggest that birds trained with the penalty-time procedure review the trial stimuli following an incorrect choice.
\end{abstract}

The two-choice matching-to-sample (MTS) task has been used extensively to assess concept learning in pigeons (Cumming \& Berryman, 1961; Zentall \& Hogan, 1974, 1976, 1978; Zentall, Edwards, Moore, \& Hogan, 1981). MTS may also be used to assess memory processes by inserting a delay between the offset of the sample and the onset of the comparison stimuli (Roberts \& Grant, 1976; Zentall \& Hogan, 1977).

One factor that may affect MTS acquisition rates is the extent to which the pigeons must both learn to respond to the comparison that is correct and learn not to respond to the comparison that is incorrect (Urcuioli \& Nevin, 1975; Zentall \& Hogan, 1978; Zentall, Hogan, \& Edwards, 1980). Urcuioli and Nevin used a training procedure in which responses to the sample produced one of the comparisons. When the comparison did not match the sample, the pigeon could turn it off and produce a matching stimulus on the other comparison key by not responding to the comparison for $4.8 \mathrm{sec}$. A response to the odd comparison reset the 4.8-sec interval. This procedure ensured that the pigeons learned what to do in the presence of both the correct and the incorrect comparison for each sample.

Zentall and Hogan (1978) trained pigeons to a high level of performance on MTS and the related oddity-from-

This research was supported by National Science Foundation Grant BNS-8418275 to Thomas R. Zentall and Peter J. Urcuioli and by a grant from the University of Kentucky Research Foundation. We thank Janice N. Steirn for her helpful comments on the manuscript. Correspondence concerning this research should be sent to Thomas R. Zentall, Department of Psychology, University of Kentucky, Lexington, KY 40506. sample (OFS) tasks. Negative instance trials, in which no correct alternative was presented, were then interspersed among the MTS or OFS trials. In the oddity task, both comparison stimuli matched the sample, whereas, in the matching task, neither comparison matched the sample. The comparison stimuli on negative instance trials were presented for a minimum of $3 \mathrm{sec}$. The trial ended when $3 \mathrm{sec}$ elapsed without a response. On these trials, the "penalty" for responding encouraged subjects to examine both choice stimuli in order to identify the negative instance trials and withhold responding to both comparisons.

Zentall et al. (1980) interspersed such negative instance trials from the start of training on a standard oddity task in which the odd stimulus could appear on any of the three response keys. Facilitated acquisition was found relative to a control group without negative instance training. Zentall et al. advanced three possible explanations for this finding. First, negative instance training may have better defined the boundary conditions of the oddity concept by forcing birds to learn not to respond to certain exemplars. Second, negative instance training may have promoted observation of all three keys before responding. Thus, errors due to impulsive responding may have been reduced. Third, negative instance trials may have made the odd stimulus more perceptually salient during regular trials after being absent on some nonreinforced trials. One problem in determining the effect of negative instance training on acquisition is that the negative instance procedure involves added training trials. Performance might be facilitated to the same degree by an equal number of added matching or oddity trials (Zentall et al., 1980). 


\section{EXPERIMENT 1}

Perhaps some features of the negative instance trials can be incorporated into standard matching trials. It may then be possible to separate the consequence of a response on a negative instance trial from the negative instance trial itself. The purpose of Experiment 1 was to assess the effects of one feature of negative instance trials-maintenance of the trial stimuli after incorrect comparison choiceson acquisition of simultaneous matching and oddity tasks. We will refer to postchoice stimulus maintenance on trials with an error as the penalty-time procedure.

\section{Method}

\section{Subjects}

Sixteen male, experimentally naive, 9-month-old White Carneaux pigeons obtained from the Palmetto Pigeon Plant, Sumter, SC, were individually housed and were maintained at approximately $80 \%$ of their free-feeding weights. Free access to water and grit were provided in the home cage. The lights were on in the colony room from $0600-1800 \mathrm{~h}$ daily.

\section{Apparatus}

Experimental sessions were conducted in a standard (LVE/BRS) pigeon test chamber, with inside measurements of $35 \mathrm{~cm}$ high, $30 \mathrm{~cm}$ wide, and $35 \mathrm{~cm}$ across the intelligence panel. The bottom edge of a centrally rear-mounted grain feeder was $9.5 \mathrm{~cm}$ from the floor. When the grain feeder was raised, it was illuminated by a 28-V, .04-A lamp. Mounted $25 \mathrm{~cm}$ above the floor was a horizontal row of three round pecking keys, $2.5 \mathrm{~cm}$ in diameter and $8 \mathrm{~cm}$ center to center. Behind each pecking key was a 12-stimulus inline projector (Industrial Electronics Engineering). Each projector could illuminate its corresponding key with a red or a green field (Kodak Wratten Filters Nos. 26 and 60, respectively), with a white annulus (16 $\mathrm{mm}$ outside diameter, $13 \mathrm{~mm}$ inside diameter), or a plus sign (made up of lines $18 \mathrm{~mm}$ long and $3 \mathrm{~mm}$ wide) on a dark background. A dim, continuously lit houselight was centered on the intelligence panel above the pecking keys. Masking of sounds was provided by white noise and an exhaust fan. Control equipment was located in an adjoining room.

\section{Procedure}

Pretraining. All birds were trained to eat from the grain feeder and then were trained to peck the circle (half of the birds) or the plus sign (the remaining birds) on the center key using an autoshaping procedure in which a $22-\mathrm{sec}$ intertrial interval (ITI) was followed by an 8 -sec stimulus presentation. As the stimulus was terminated, the grain feeder was raised for $5 \mathrm{sec}$. Following reinforcement, the next ITI began. If a response was made to the center key during stimulus presentation, the stimulus was immediately terminated and reinforcement was made available. Responses made to the center key during the intertrial interval or reinforcement had no programmed consequences. Pretraining continued until a response was made to each of eight or more consecutive stimulus presentations. When this criterion was reached, the number of responses required for reinforcement was gradually increased to 10 .

Training. The subjects were randomly assigned to four groups, with the constraint that half of the subjects in each group had been pretrained with a plus sign and half had been trained with a circle. The subjects in all groups were given daily sessions of 96 trials. Trials began with the presentation of a sample stimulus on the center key. Trials were presented in random order, with the constraint that a stimulus could occur as the sample on no more than three consecutive trials. Following the 10th response to the sample key, stimuli were presented on the side (comparison) keys (the sample remained lit). One of the comparisons matched the sample, and the other was the alternative (odd) training stimulus. For the birds in Groups Oddity Penalty (OP) and Oddity Control (OC), initial responses to the odd comparison key were reinforced, whereas, for the birds in Groups Matching Penalty (MP) and Matching Control (MC), a choice response to the matching comparison was reinforced. A correct response by the birds in Groups $\mathrm{MC}$ and $\mathrm{OC}$ was immediately rewarded by the offset of all stimuli and access to the grain feeder for $2.5 \mathrm{sec}$ of the 5 -sec ITI. An incorrect side-key response resulted in the offset of all stimuli and initiation of the ITI. Additional pecks to the sample had no programmed consequence.

The consequences of correct responding by Groups MP and OP were the same as those by the control groups. However, an initial comparison-key response to the incorrect comparison resulted in penalty time for Groups MP and OP, but not for the control groups. Penalty time consisted of continued presentation of the training stimuli for $4 \mathrm{sec}$ in the absence of further responding. Responses made to any of the three response keys during the penalty time had the effect of resetting the 4 -sec penalty-time clock. Thus, following an incorrect response, $4 \mathrm{sec}$ of penalty time had to elapse without a response before the display was terminated and the ITI began.

There were 36 training sessions. The primary measure of performance was percentage correct trials per session. In addition, mean comparison choice latency was assessed for each bird on each session.

\section{Results}

Two birds failed to break from chance within 24 training sessions, one each from Groups $O P$ and $O C$. These two birds were dropped from the study, and their data do not appear in any of the data analyses.

Acquisition of the matching and oddity tasks was faster for the penalty-time groups than for the control groups. One measure of early acquisition is the first session in which each pigeon performed at a level that exceeded and remained above chance ( $50 \%$ correct) on succeeding sessions. A two-way analysis of variance performed on these data indicated that penalty-time birds reached this criterion earlier in training (mean $=6.3$ sessions) than did control birds (mean $=11.0$ sessions) $[F(1,10)=7.96]$. The .05 level of significance was used for this and all subsequent analyses. However, neither the main effect of task (matching vs. oddity) nor the task $\times$ penalty time versus control interaction was significant (both $F \mathrm{~s}<1$ ).

A similar analysis performed on the sessions-to- $90 \%$ scores (first session at or above $90 \%$ correct) also indicated that the penalty-time birds reached criterion significantly faster (mean $=11.1$ sessions) than did the control birds (mean $=16.8$ sessions) $[F(1,10)=5.10]$. Again, neither the main effect of task nor the task $\times$ penalty time versus control interaction was significant.

Another analysis, performed on percentage correct performance pooled over the first 16 sessions of training, also indicated a significant effect of penalty time $[F(1,10)=$ 28.11], but no other significant effects. Acquisition functions for the four groups appear in Figure 1.

Examination of choice latency data indicated that, for each bird, choice latency systematically increased and then decreased with training. Furthermore, the peak value of choice latency for each bird occurred at or about the first session at which performance consistently exceeded chance. A Pearson product-moment correlation revealed 


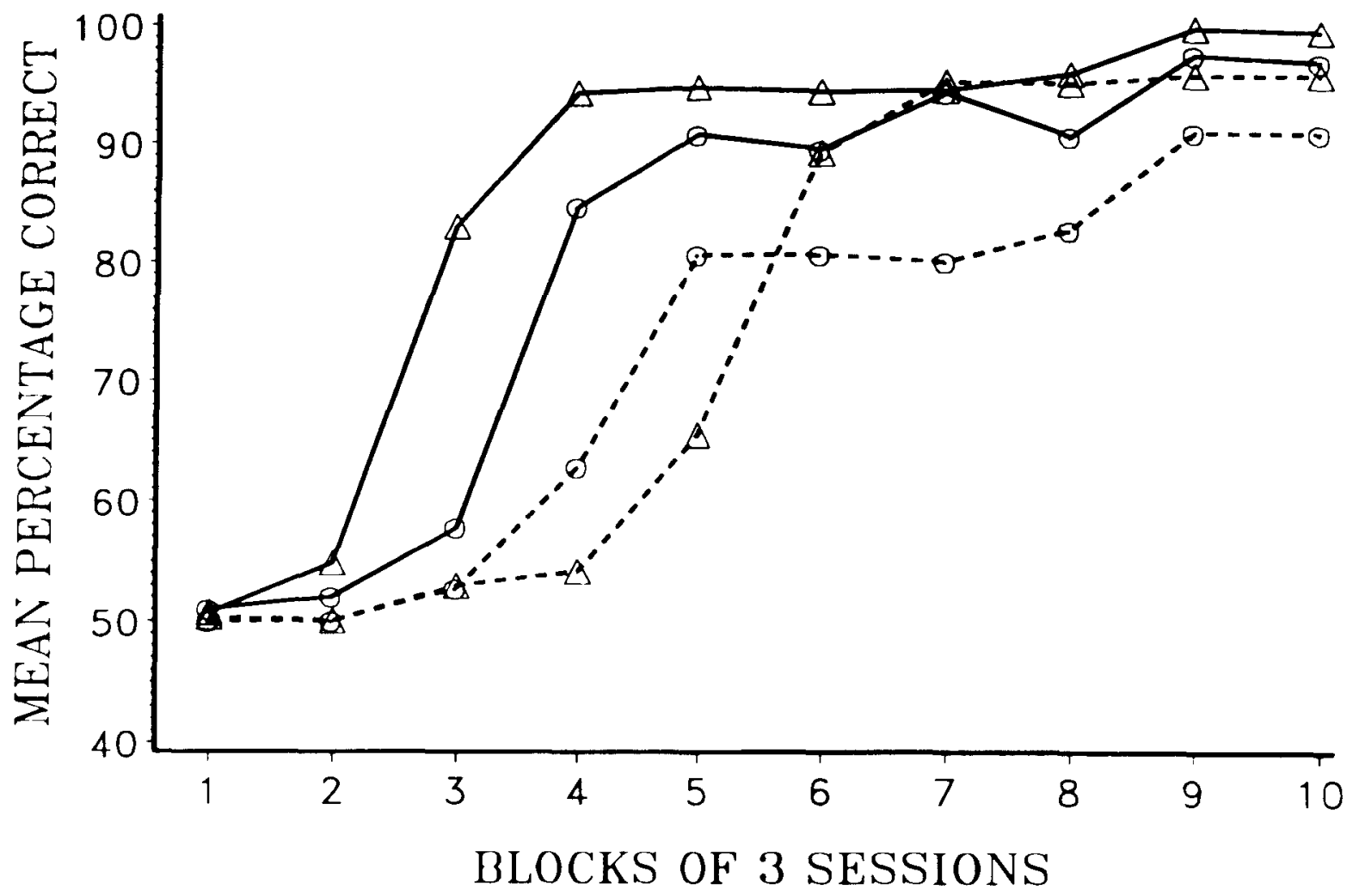

Figure 1. Acquisition functions for penalty-time (solid lines) and control (dashed lines) groups trained on matching-to-sample (circles) and oddity-from-sample (triangles) in Experiment 1.

a significant relation between the session on which the choice latency peaked and the first session on which performance exceeded chance $[r(14)=0.95]$.

Examination of the peak values indicated that the mean peak latency per trial was longer for the penalty groups (mean $=8.45 \mathrm{sec}$ ) than for the control groups (mean = $2.07 \mathrm{sec}$ ). Because the distribution of peak latencies was highly (positively) skewed, these scores were subjected to a square root transformation, and a two-way analysis of variance was performed. The analysis indicated that there was a significant effect of penalty time $[F(1,10)=$ 8.05]. No other effect was significant. Choice-response latency data, plotted relative to the first session that performance exceeded and remained above $50 \%$ correct (for each bird), appears in Figure 2.

\section{Discussion}

The results of Experiment 1 indicate that a penalty-time procedure can significantly facilitate acquisition of MTS and OFS tasks. Both penalty-time and negative-instance trials feature maintenance of the trial stimuli after a nonreinforced trial response and termination of the trial contingent upon no further responses to the maintained stimuli. One way in which penalty-time and negativeinstance training might enhance learning is by motivating the animal to lengthen the time spent examining the trial stimuli before making the choice response. The firstpeck latency data suggest that improved discrimination performance may accompany an increase in choice latency. Perhaps any procedure that produces an increase in choice latency would result in faster task acquisition.

A direct test of the effects of an enforced choice-point pause was made by Muenzinger and Fletcher (1937), who inserted glass doors at the choice point of a $\mathrm{T}$-maze and required rats to pause for $5 \mathrm{sec}$ prior to making their choices. Rats that received the enforced delay made fewer errors and reached a learning criterion in fewer trials than did controls that received no delay. A choice-point pause also can be produced by presentation of an aversive event (e.g., shock) just after the choice response (Muenzinger $\&$ Wood, 1935). Such a pause is also associated with faster discrimination learning. In both the penalty-time and the negative-instance trial procedures, the stimulus keys remain lit for a minimum amount of time and additional responding delays termination of the trial. This contingency may be aversive, because pigeons tend to make a large number of nonreinforced responses to the comparison stimuli and because the presentation of a new trial (and reinforcement opportunity) is delayed.

Increased choice latency may facilitate performance by discouraging impulsive responses to the comparison keys (Wilkie \& Spetch, 1981). Perhaps on some trials pigeons make elicited responses to a comparison key before ex- 


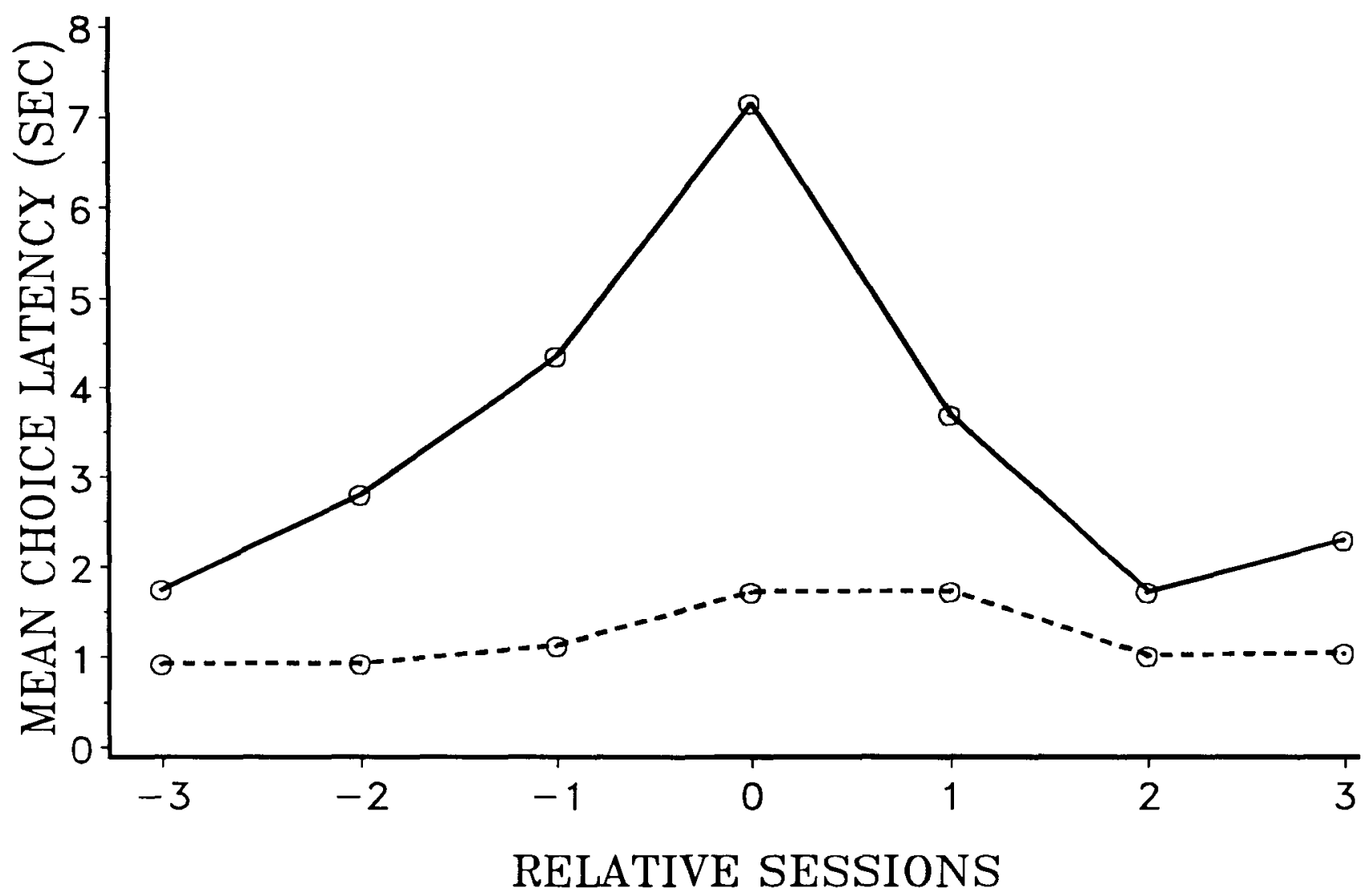

Figure 2. Choice-response latencies for penalty-time (solid lines) and control (dashed lines) groups, plotted by session relative to the first session that performance exceeded and remained above chance (50\% correct) in Experiment 1.

amining it. In the present experiment, this is especially likely during early sessions of training, after pretraining with an autoshaping procedure that involved no discrimination. Elicited responding in a two-choice procedure would be maintained by reinforcement on $50 \%$ of the trials.

\section{EXPERIMENT 2}

If the penalty-time procedure facilitates acquisition of the matching and oddity tasks by increasing choiceresponse latency, then facilitation should also be observed when choice latency is increased by other procedures. One way to increase choice latency is to delay the effective choice response by making comparison responses ineffective for a short time following the onset of the comparison keys. Such a schedule of comparison-key pecking is essentially a discrete-trial fixed-interval (FI) schedule.

In a preliminary study, an FI 2 -sec schedule imposed on the matching and oddity tasks had no effect on acquisition, as compared with a control group trained without the FI contingency. Although time of exposure to the comparison stimuli was increased, the desired effect of slowed comparison-key pecking was not produced. Instead, the subjects responded steadily and vigorously throughout the 2-sec interval. The apparent lack of delay in making the comparison choice may have been due to the very short duration of the interval, or perhaps a delay failed to occur because the subjects were trained to respond to the sample on a FR schedule.

The FI schedule was introduced to discourage choices immediately after comparison onset. Another way to accomplish this goal would be to require the absence of responding during the first $2 \mathrm{sec}$ of comparison presentation by introducing what is essentially a schedule involving discrete-trial differential reinforcement of low rates of responding (i.e., a DRL schedule of responding for trial termination). With this schedule in effect, responses made on any of the three keys during the first $2 \mathrm{sec}$ of comparison stimulus presentation would reset the DRL timer. Thus, trial termination would occur with the first comparison response after $2 \mathrm{sec}$ had elapsed without a response. The purpose of Experiment 2 was to determine whether such an enforced pause is effective in facilitating choice performance.

\section{Method}

\section{Subjects and Apparatus}

The subjects were 16 experimentally naive, 9-month-old, male White Carneaux pigeons housed and maintained as described in Experiment 1. The apparatus and stimuli were as described in Experiment 1 . 


\section{Procedure}

Pretraining and Training. Pretraining was as described in Experiment 1 , but in addition, the pigeons were given 15 presentations of the pretraining stimulus to each of the side keys to encourage side-key responding. Each pigeon was then assigned to one of the four groups, as they were in Experiment 1. The groups included matching control (MC) and oddity control (OC) groups, which were trained as described in Experiment 1, and matching DRL (MDRL) and oddity DRL (ODRL) groups, which were trained on the matching or oddity tasks in which trial terminating comparison choices involved a DRL 2 -sec schedule.

For the DRL groups, responses made to any of the training stimuli during the first $2 \mathrm{sec}$ of comparison presentation had the effect of resetting the DRL timer. An additional $2 \mathrm{sec}$ then had to elapse before a comparison-key peck could terminate the trial. Correct trial-terminating responses were reinforced with 2.5 -sec access to mixed grain. Incorrect trial-terminating responses ended the trial without reinforcement. In all other respects, sessions were as described in Experiment 1. All birds were given 30 sessions of training.

\section{Results}

The performance of one of the MC birds and one of the MDRL birds never rose consistently above chance. Their data do not appear in any of the analyses. Acquisition functions for the birds in the four training groups appear in Figure 3.

The data in Figure 3 suggest a possible effect of choice schedule (DRL vs. control), and perhaps also an effect of matching versus oddity. However, two-way analyses of variance involving three different performance measures (sessions to break from and remain above chance, sessions to the first session at or above $60 \%$ correct, and asymptotic level of performance-i.e., performance on the last three training sessions) indicated that there were no significant main effects or interactions.

Latency to the first choice response on each trial, regardless of its consequence, was also examined. Analysis of cumulative latencies, pooled over all sessions, indicated that the DRL schedule produced a significant increase in the latency of the initial choice of a comparison stimulus $[F(1,12)=264.16]$.

\section{Discussion}

As expected, the DRL contingency increased the latency of the first comparison-key response. However, the enforced choice-point pause did not facilitate matching or oddity learning as it apparently did for Muenzinger and Fletcher (1937).

The DRL schedule encourages pigeons to wait before making a choice response. The purpose of delaying the choice response was to prevent incorrect elicited responses from being counted and to allow the pigeon to examine the stimulus array prior to making a choice. Unfortunately, there was no programmed contingency in the schedule that

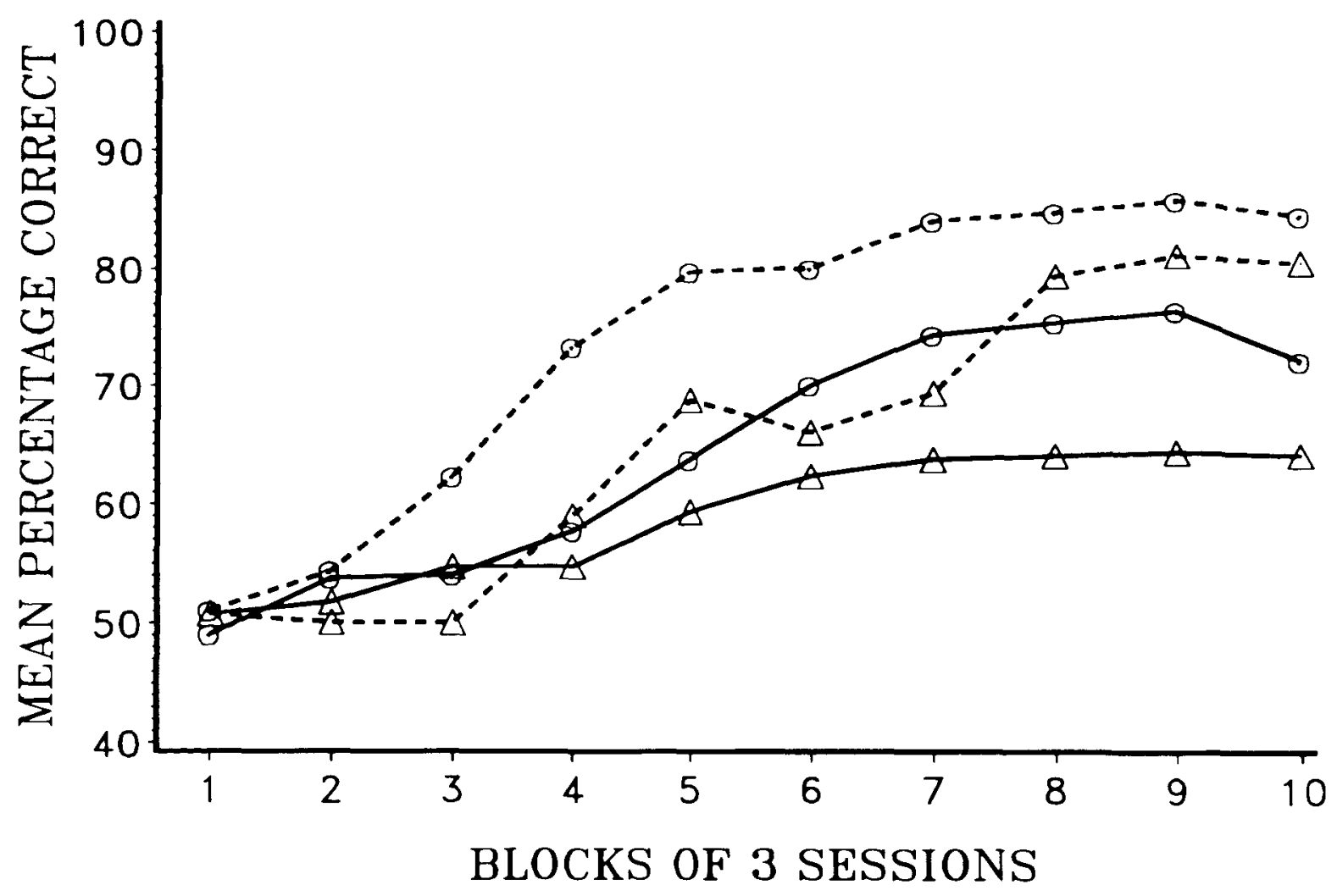

Figure 3. Acquisition functions for DRL comparison contingency (solid lines) and control (dashed lines) groups trained on matching-to-sample (circles) and oddity-from-sample (triangles) in Experiment 2. 
required the pigeons to examine the stimuli, and during the interval before the choice, the pigeons may have engaged in behavior unrelated to stimulus observation. In other experiments, pigeons reinforced on a DRL schedule often show stereotyped collateral behavior that may serve to time the delay interval (Ferster \& Skinner, 1957; Nevin $\&$ Berryman, 1963). Furthermore, pecking during the interval is punished by increasing the interval length, perhaps causing the comparison stimuli to become aversive.

Although increased comparison response latency accompanied the start of improved matching and oddity performance in Experiment 1, the data from Experiment 2 indicate that a significant increase in choice latency alone is not sufficient to facilitate matching or oddity learning. It is unlikely that this factor alone underlies the effects of penalty-time and negative-instance training.

\section{EXPERIMENT 3}

Reduced probability of elicited pecks produced by an increase in choice delay is not the only mechanism by which the penalty-time procedure might facilitate matching acquisition. Three additional mechanisms can be identified: (1) penalty time may increase the discriminability of the outcomes of correct and incorrect responding, (2) the aversiveness of trial prolongation following an incorrect response may increase the motivation to avoid making errors, and (3) penalty time may provide an opportunity for the subject to review the trial stimuli following an incorrect response.

In matching and oddity procedures typically used with pigeons, the only difference in the outcomes of correct versus incorrect choice responses is the occurrence of reinforcement or nonreinforcement. According to an information interpretation of reinforcement (e.g., Estes, 1969), the information content of the outcomes can be increased by the addition of predictable salient events occurring differentially after correct and incorrect responses. In the case of penalty-time training, an incorrect response results in an added stimulus event and response contingency (trial maintenance) not associated with correct responding. Discrimination performance may improve with penalty-time training because the internal representations of the outcomes following correct versus incorrect responses are more discriminable for these animals (Peterson \& Trapold, 1980).

The results of several studies (e.g., Brodigan \& Peterson, 1976; Zentall, Hogan, \& Edwards, 1984) indicate that matching in the pigeon may involve the association of trial stimuli with internal representations of trial outcomes. Differential outcomes have been found to facilitate acquisition (Peterson, 1984) and transfer (Edwards, Jagielo, Zentall, \& Hogan, 1982) of symbolic matchingto-sample tasks (i.e., tasks in which the sample and comparison stimuli come from orthogonal dimensions-e.g., hue samples and line comparisons) in pigeons.

A second way in which penalty-time training may facilitate task acquisition is by increasing the pigeon's motivation to avoid making errors. Prolongation of the trial by delaying the start of the ITI following an incorrect response may be sufficiently aversive to significantly increase the differential consequences of correct and incorrect responding.

A third mechanism by which penalty-time training may facilitate learning is by providing an opportunity for the subject to review the trial stimuli following an incorrect choice response (stimulus feedback). The major difference between this explanation and the previous two is that an increase in the differentiation of outcomes can be produced by any increase in discriminability of events occurring after choice, whereas stimulus feedback specifically involves the maintenance of trial stimuli (i.e., trialspecific information following a choice response).

Trial stimuli have been maintained in a T-maze brightness discrimination by providing a rat with reinforcement in a goalbox of the same brightness as the correct arm of the maze. Groups trained in this way learned significantly faster than did controls that received reinforcement in the presence of a brightness different from that of the correct arm (Eninger, 1953). In a human concept-identification study, Bourne, Guy, Dodd, and Justesen (1965) found a general facilitation of performance due to trial-stimulus maintenance. Trial-stimulus maintenance may protect against forgetting of the trial stimuli and thus may allow those stimuli to be better associated with the trial outcome.

In Experiment 3, the contributions of trial prolongation, increased differential outcomes, and postchoice stimulus review were assessed. Experiment 3 involved four groups, each exposed to matching trials. The first group, Group PEN, was trained with penalty time, similar to that for Group MP in Experiment 1. For this group, incorrect responses resulted in a minimum of 4-sec maintenance of trial stimuli. The second group, Group WPEN, was exposed to a penalty-time procedure, but the penalty interval was spent in the presence of white keys. This group served as a control for trial prolongation following an incorrect response. The third group, Group WITI, did not have a penalty contingency, but the keys were white or dark during the ITI depending on whether the response was correct or incorrect. This group had a differential outcome for correct and incorrect responses that was similar to that of the WPEN group, but it provided a baseline against which to assess the effect of error-produced delay in the start of the ITI (i.e., for Group WITI, errors did not prolong the trial). Finally, the fourth group, Group CONT, was trained with a standard matching task, similar to that for Group MC in Experiment 1. This group did not have differential key lights during the ITI; thus, it allowed for assessment of the effect of an additional differential outcome provided during the ITI.

According to the outcome-discriminability hypothesis, the addition of differential postresponse cues makes the outcomes of correct and incorrect responding more discriminable. Thus, the added differential outcomes associated with incorrect responding in Groups PEN, WPEN, and WITI should facilitate acquisition, relative to that in Group CONT.

On the other hand, trial prolongation per se may be sufficient to account for facilitated acquisition by birds trained with the penalty-time procedure. With penalty time, not 
only are incorrect responses followed by the absence of reinforcement, but they also result in a delay in the start of the ITI and, thus, a delay in the start of the next trial. Perhaps trial prolongation is sufficiently aversive to motivate a reduction in comparison-choice errors. If so, then Groups PEN and WPEN should learn faster than Groups WITI and CONT.

Finally, if postchoice stimulus processing is responsible for facilitated learning by birds trained with the penaltytime procedure, then Group PEN should learn faster than the other three groups, because only penalty-time birds have the opportunity to review trial stimuli after the choice response has been made. This result would indicate that the maintained stimuli are actively processed following an incorrect response.

\section{Method \\ Subjects \\ Sixteen unsexed White Carneaux pigeons, 5-8 years old, were obtained from Palmetto Pigeon Plant and maintained as described in Experiment 1.}

\section{Apparatus}

The apparatus was the same as in Experiment 1, except for the training stimuli that could be projected onto the three keys. The new training stimuli were a single white vertical line and a single white horizontal line on a dark background, as well as a white uniform field.

\section{Procedure}

Pretraining and Training. All birds were pretrained as described in Experiment 2 and were then randomly assigned to one of four groups, according to the procedure described in Experiment 1. Groups CONT and PEN were treated similarly to Groups $\mathrm{MC}$ and MP, respectively, in Experiment 1, except that 15 responses to the sample key were required to produce the comparisons. The sampleresponse ratio was increased to encourage all birds to learn their tasks.

Training for Group WPEN was identical to that of Group PEN, except that the stimulus keys were white during the penalty interval following a response to the incorrect (odd) comparison.

Training for Group WITI was identical to that for controls except that, following a choice response to the incorrect (odd) comparison, a white field was projected onto all three response keys. The keys remained white through the ITI. Responding to the white keys during the intertrial interval had no programmed consequences. All subjects were trained to a criterion of $90 \%$ correct over the trials in a session.

\section{Results and Discussion}

Matching acquisition was somewhat faster in Experiment 3 than in Experiments 1 and 2. Overall, the pigeons acquired the matching task to a criterion of $90 \%$ correct in an average of 11.7 sessions (as compared with 16.8 sessions in Experiment 1). Faster acquisition was probably due to the increase in sample-stimulus response requirement (from 10 in Experiments 1 and 2 to 15 in Experiment 3), though the change in sample stimuli (from circles and plus signs in Experiments 1 and 2 to vertical and horizontal lines in Experiment 3) may have con- tributed as well. Acquisition functions for the four training groups appear in Figure 4.

A one-way analysis of variance performed on sessionsto-criterion (first session $\geq 90 \%$ correct) scores indicated that there was a significant group effect $[F(3,12)=4.89]$. Planned comparisons involving the four groups indicated that (1) Group PEN acquired the matching task significantly faster (mean $=9.5$ sessions) than the other three groups combined (mean $=18.2$ sessions) $[F(1,12)=$ 11.53], (2) Group WPEN did not acquire the task significantly faster (mean $=18.00$ sessions) than Groups WITI and CONT combined (mean $=18.2$ sessions) $(F<1)$, and (3) Group WITI did not acquire the task significantly faster (mean $=16.3$ sessions) than Group CONT (mean = 20.2 sessions) $[F(1,12)=1.97]$.

A similar set of analyses was performed on the percentage correct data pooled over the first eight sessions (all birds completed at least eight sessions). Again, the one-way analysis indicated a significant group effect $[F(1,12)=6.75]$. Again, planned comparisons indicated that Group PEN performed at a higher level than the other three groups $[F(1,12)=17.64]$, but that performance among the other groups did not differ significantly. Furthermore, a post hoc (least significant difference) comparison indicated that performance by Group WPEN did not differ significantly from that of Group WITI $[F(1,12)=$ 2.18].

The results of Experiment 3 suggest that stimulus feedback is the major factor in producing the penalty-time effect (Group PEN vs. Groups WPEN, WITI, and CONT). The added aversive consequences of extending the trial following an incorrect response (Group WPEN vs. Groups WITI and CONT) apparently had little effect on acquisition.

\section{GENERAL DISCUSSION}

The results of Experiments 1 and 3 indicate that the penalty-time procedure (involving maintenance of trial stimuli for $4 \mathrm{sec}$ without a response following an incorrect comparison choice) results in faster acquisition of simultaneous matching and oddity tasks. At a practical level, these data suggest that penalty-time procedures should be incorporated into any conditional discrimination task in which rapid acquisition is desired (see Riley \& Roitblat, 1978, p. 251).

In Experiment 1, the significantly longer choice time found for the birds trained with the penalty-time procedure, as compared with controls, suggested that a delay in choice may be responsible for facilitated acquisition.

However, in Experiment 2, when choice-response time was increased by requiring the birds to pause $2 \mathrm{sec}$ before making their choice responses (i.e., the DRL contingency when trial termination occurred following the first choice response after $2 \mathrm{sec}$ had elapsed), no facilitation was found. Thus, the increase in choice-response time, per se, is probably not responsible for facilitated acqui- 


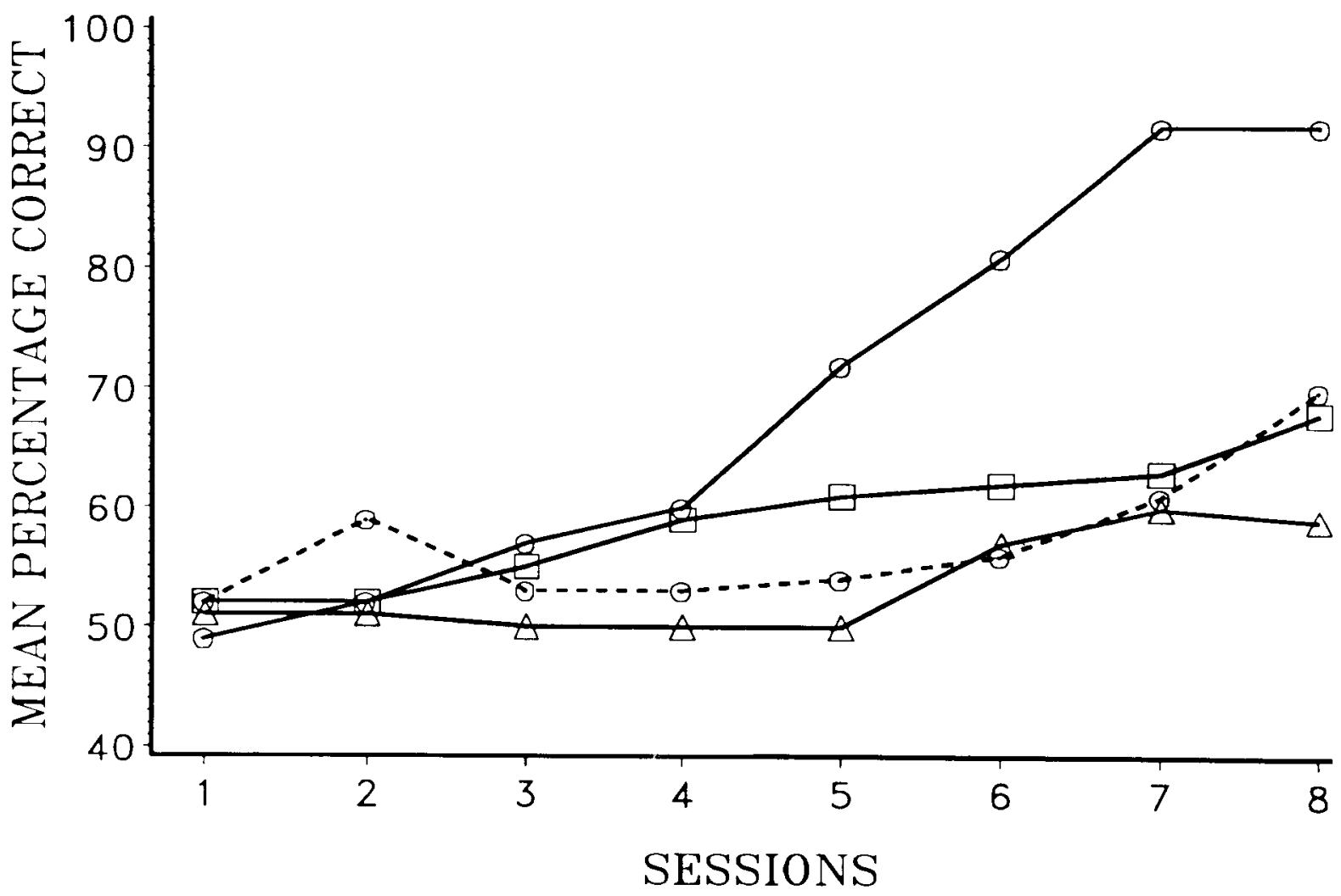

Figure 4. Acquisition functions for penalty-time (PEN; circles, solid lines), white penalty-time (WPEN; triangles, solid lines), white intertrial interval (WTTI; squares, solid lines), and control (CONT; circles, dashed lines) groups in Experiment 3.

sition. It is possible that some other procedural manipulation that produces an increase in choice-response time would result in facilitated acquisition, but neither the DRL nor the FI comparison-response contingency appear to produce an effect comparable to penalty time.

Nevertheless, the finding that the peak value of choice latency coincided almost perfectly with the first session that performance was better than chance (see Figure 2) suggests that choice latency is somehow related to task acquisition.

The results of Experiment 3 suggest that the mechanism responsible for facilitated acquisition with the penalty-time procedure is not an increase in discriminability of consequences following an incorrect response, nor is it the aversive effects of trial prolongation. Instead, the results suggest that, following an incorrect response, maintenance of the stimulus display serves to maintain the conditions that led to the incorrect response and allows the pigeon to review the trial stimuli.

If postchoice stimulus review is responsible for facilitated acquisition, one would expect this facilitation to be eliminated or greatly reduced by training on a zero-delay matching task (onset of the comparison stimuli is accompanied by offset of the sample). Similarly, one would expect less facilitation if, following half of the incorrect comparison responses on a simultaneous matching task, the sample stimulus was replaced by the alternate sample. If the pigeon uses delayed offset of the trial stimuli to review sample and comparison events, sample-replacement trials should provide inappropriate feedback and performance should suffer.

Such findings would support the contention that pigeons can extract trial information following a choice response and that they have information-processing capacity that has not previously been acknowledged.

\section{REFERENCES}

Bourne, L. E., JR., GuY, D. E., DodD, D. H., Justesen, D. R (1965). Concept identification: The effects of varying length and informational components of the intertrial interval. Joumal of Experimental Psychology, 69, 624-629.

Brodigan, D. L., \& Peterson, G. B. (1976). Two-choice conditional discrimination performance of pigeons as a function of reward expectancy, prechoice delay, and domesticity. Animal Learning \& Behavior, 4, 121-124.

Cumming, W. W., Berryman, R. (1961). Some data on matching behavior in the pigeon. Joumal of the Experimental Analysis of Behavior, 4, 281-284.

Edwards, C. A., Jagielo, J. A., Zentall, T. R., \& Hogan, D. E. (1982). Acquired equivalence and distinctiveness in matching to sample by pigeons: Mediation by reinforcer-specific expectancies. Journal of Experimental Psychology: Animal Behavior Processes, 8, 244-259.

ENINGER, M. U. (1953). The role of generalized approach and avoidance tendencies in brightness discrimination. Journal of Comparative \& Physiological Psychology, 46, 398-402.

Estes, W. K. (1969). Reinforcement in human learning. In J. Tapp 
(Ed.), Punishment and aversive behavior (pp. 63-94). New York: Appleton-Century-Crofts.

FERSTER, C. B., SkINNER, B. F. (1957). Schedules of reinforcement. New York: Appleton-Century-Crofts.

Muenzinger, K. F., \& Fletcher, F. M. (1937). Motivation in learning: VII. The effect of an enforced delay at the point of choice in the visual discrimination habit. Journal of Comparative Psychology, 23, 383-392.

Muenzinger, K. F., \& WoOd, A. (1935). Motivation in learning: IV. The function of punishment as determined by its temporal relation to the act of choice in the visual discrimination habit. Journal of Comparative Psychology, 20, 95-106.

NEVIN, J. A., BeRRYMAN, R. (1963). A note on chaining and temporal discrimination. Joumal of the Experimental Analysis of Behavior, 6, 109-113.

Peterson, G. B. (1984). How expectancies guide behavior. In H. L. Roitblat, T. G. Bever, \& H. S. Terrace (Eds.), Animal cognition (pp. 135-148). Hillsdale, NJ: Erlbaum.

Peterson, G. B., \& Trapold, M. A. (1980). Effects of altering outcome expectancies on pigeons' delayed conditional discrimination performance. Learming \& Motivation, 11, 267-288.

Riley, D. A., Roitblat, H. L. (1978). Selective attention and related cognitive processes in pigeons. In S. H. Hulse, H. Fowler, \& W. K. Honig (Eds.), Cognitive processes in animal behavior (pp. 249276). Hillsdale, NJ: Erlbaum.

Roberts, W. A., \& Grant, D. S. (1976). Studies of short-term memory in the pigeon using the delayed matching to sample procedure. In D. L. Medin, W. A. Roberts, \& R. T. Davis (Eds.), Processes of animal memory (pp. 79-112). Hillsdale, NJ: Erlbaum.

Urcuioli, P. J., Nevin, J. A. (1975). Transfer of hue matching in pigeons. Joumal of the Experimental Analysis of Behavior, 24, 149-155.

Wilkie, D. M., SPETch, M. L. (1981). Pigeons' delayed matching to sample errors are not always due to forgetting. Behaviour Analysis Letters, 1, 317-323.

Zentall, T. R., Edwards, C. A., Moore, B. S., \& Hogan, D. E. (1981). Identity: The basis for both matching and oddity learning in pigeons. Journal of Experimental Psychology: Animal Behavior Processes, 7, 70-86.

Zentall, T. R., Hogan, D. E. (1974). Abstract concept learning in the pigeon. Journal of Experimental Psychology, 102, 393-398.

Zentall, T. R., Hogan, D. E. (1976). Pigeons can leam identity or difference, or both. Science, 191, 408-409.

ZENTALL, T. R., HoGAN, D. E. (1977). Short-term proactive inhibition in the pigeon. Learning \& Motivation, 8, 367-386.

Zentall, T. R., Hogan, D. E. (1978). Same/different concept learning in the pigeon: The effect of negative instances and prior adaptation to the transfer stimuli. Journal of the Experimental Analysis of Behavior, 30, 177-186.

Zentall, T. R., Hogan, D. E., Edwards, C. A. (1980). Oddity learning in the pigeon: Effect of negative instances, correction, and number of incorrect alternatives. Animal Learning \& Behavior, 8 , 621-629.

Zentall, T. R., Hogan, D. E., e Edwards, C. A. (1984). Cognitive factors in conditional learning by pigeons. In $\mathrm{H}$. L . Roitblat, T. G. Bever, \& H. S. Terrace (Eds.), Animal cognition (pp. 389-405). Hillsdale, NJ: Erlbaum.

(Manuscript received June 20, 1990; revision accepted for publication October 9,1990 .) 